\title{
Retrospective drug utilization review: impact of pharmacist interventions on physician prescribing
}

This article was published in the following Dove Press journal:

ClinicoEconomics and Outcomes Research

24 June 2011

Number of times this article has been viewed

\section{Mallik Angalakuditi' Joseph Gomes ${ }^{2}$}

'Georgia State University, Atlanta, GA, USA; 'Baxter Health Care, Deerfield, IL, USA
Correspondence: Mallik Angalakuditi

Georgia State University,

Atlanta, GA, USA

Tel +l 20I 6558662

Email hydmallik02@hotmail.com
Objectives: To evaluate the impact of retrospective drug utilization review (RDUR), pharmacist's interventions on physician prescribing, and the level of spillover effect on future prescriptions following the intervention.

Methods: A retrospective case-control study was conducted at a pharmacy benefits management company using the available prescription data from April 2004 to August 2005. RDUR conflicts evaluated and intervened by a clinical pharmacist served as a case group, whereas conflicts that were not evaluated and intervened by a clinical pharmacist served as a control group.

Results: A total of 40,284 conflicts in cases and 13,044 in controls were identified. For cases, 32,780 interventions were considered nonrepetitive, and 529 were repetitive. There were 22,870 physicians in cases that received intervention letters and 2348 physicians in the control group that would have received intervention letters during the study period. Each physician received on average 1.4 interventions for cases vs 3.0 for controls. Among the case physicians who were intervened during the study period, $2.2 \%(505)$ were involved in a repeated intervention vs $18.2 \%$ (428) in controls $(P<0.001)$, which is an eight-fold difference. The most common conflict intervened on in cases was therapeutic appropriateness $(8277,25.3 \%)$, and for controls it was drug-drug interactions $(1796,25.4 \%)$. The overall interventional spillover effect in cases was $98.4 \%$ vs $89.4 \%$ in controls $(P=0.01)$.

Conclusion: RDUR is an effective interventional program which results in decreased numbers of interventions per physician and provides a significant impact on future prescribing habits.

Keywords: pharmacy management, spillover effect, RDUR, DUR

\section{Introduction}

The introduction of pharmacy benefits management companies came about due to the need for a point-of-service system to adjudicate pharmacy claims in the 1980s. Over the years, other value-added programs such as drug usage review (DUR), generic substitution, and step-care protocols have been added to improve quality of medical care and control health care costs. According to the 2006 Novartis Pharmacy Benefit Report, ${ }^{1}$ services such as DUR and generic substitution are now used by over $90 \%$ of the managed care organizations surveyed. The use of generic substitution and DUR is now the most frequently reported quality and cost control measure. Specifically, the use of DUR increased over the period for preferred provider organizations (2003-2004, 60.9\%-70.4\%) and health maintenance organizations (2003-2004, 68.4\%-74.4\%). It has long been recognized that drugs are not frequently used to their full potential, nor according to usually accepted criteria., ${ }^{2,3}$ Since the majority of the prescriptions are written by physicians, their prescribing habits are important when considering the inappropriateness of drug use. ${ }^{4}$ 
The goals of DUR were explained by Knapp et $\mathrm{al}^{5}$ as the encouragement of optimal drug use and the provision of high-quality drug therapy as cost-effectively as possible. Pharmacists are frequently called upon to assess medication prescribing by physicians and provide the important service of DUR. ${ }^{2}$ The outcomes of these assessments often lead to improvements in cost-effective prescribing and better utilization of limited resources. ${ }^{4}$ Although DUR is part of the vast majority of managed care quality assurance programs, the benefit of such a program has conflicting results reported in the literature.

A typical drug evaluation process generally entails an in-depth analysis of an agreed specific therapeutic group or groups. ${ }^{6}$ The method of analysis generally involves a pharmacist screening the literature and clinical data, developing and gaining agreement on practice guidelines in conjunction with other related departments, and evaluating the collected data against it. ${ }^{2}$ The results of the review will be presented to the prescribers along with medical literature and education to support the modification of their prescribing behavior. ${ }^{2}$ To ensure compliance, physician prescribing behavior is then monitored over time. DUR is a quality assurance approach for the facility per se, and it involves the setting of criteria and standards, an assessment phase using a set of screening criteria, and a follow-up correctional phase with the prescriber. ${ }^{7}$ It comprises all aspects of drug treatment from the time a patient presents to a prescriber to the final outcome of the therapy. ${ }^{7}$

The objective of this study is to evaluate a retrospective DUR (RDUR) pharmacist intervention program on physicians prescribing for adult patients. This is achieved by identifying the impact of intervention on physician prescribing and evaluating the level of spillover effect on future prescriptions following the intervention.

\section{Methods}

A retrospective case-control study was conducted at a pharmacy benefits management company (PBM) using the available prescription data from April 2004 to August 2005. The PBM has an electronic data repository that houses all pharmacy claims data. Pharmacy claims data is sent to an outside vendor, where it is evaluated against medical claims data and then returned to the PBM. A severity score is assigned to each conflict based on predetermined criteria. The clinical pharmacist then evaluates those conflicts against evidence-based guidelines and categorizes them into clinically significant and clinically nonsignificant groups. Only $1 \%$ of all clinically significant conflicts obtained from available pharmacy claims data were reviewed in detail, and interventions were made where applicable. All the intervened conflicts were included in the case group for this study. Clinically significant conflicts which were generated by the RDUR software but not intervened by a clinical pharmacist served as the control group. In the control group, pharmacist interventions were not implemented but were treated as though the intervention was implemented for the study purposes. For the purposes of this study, all case physicians who were intervened with in the study were eliminated from the control groups.

A conflict occurs when the prescription does not meet RDUR-established criteria (eg, drug-drug interactions, therapeutic appropriateness). A nonrepeated intervention is an intervention with a physician for different patients on a specific criterion that can occur multiple times within a 60-day period of the first intervention. For the purpose of this study, the nonrepetitive intervention is considered one intervention no matter how many times it occurs within 60 days. A repeated intervention is an intervention with a physician for different patients on a specific criterion that occurs 60 days after the first intervention. The physician spillover effect evaluates the impact of past interventions on prescribers for the future care of the patients. The interventional spillover effect evaluates the rate of repetitive interventions that occur based on the total number of nonrepetitive interventions.

The following data collected for the study included patient demographics, pertinent prescription information (such as drug name, date of prescription), physician ID, and intervention details. Data analyses were performed using SPSS Statistics software (v 11.5; IBM Corporation, Somers, NY). The case group was compared with the control group for spillover effect during the study period. A descriptive analysis of the case group was performed.

\section{Results}

In the case group, 40,284 conflicts were identified and of those, 32,780 interventions were considered nonrepetitive and 529 were repetitive. In the control group, 13,044 conflicts were identified, and of those, 7069 interventions were considered nonrepetitive and 748 were repetitive. The total of repetitive and nonrepetitive interventions is less than the total number of conflicts, because those conflicts that occur within 60 days after the first intervention were excluded from the analysis.

There were 22,870 physicians in cases that received intervention letters and 2348 physicians in the control group 
that would have received intervention letters during the study period. Each physician received on average 1.4 interventions for cases vs 3.0 in controls. Case physicians (14,210, 62.1\%) were more likely to receive one nonrepetitive intervention compared with controls, whereas in other categories, control physicians were more likely to receive higher numbers of nonrepetitive interventions (Table 1).

The most common conflict intervened on in cases was therapeutic appropriateness $(8277,25.3 \%)$, and for controls it was drug-drug interactions (1796, 25.4\%). Other conflict types were very similar between cases and controls (Table 2 ). The rate of repeated interventions was lower in cases vs controls. Of these 22,870 physicians who were intervened during the study period, $2.2 \%(505)$ were involved in a repeated intervention vs $18.2 \%$ (428) in controls $(P<0.001)$. The actual physician spillover effect was $16 \%(2.2 \%-18.2 \%)$.

The overall interventional spillover effect was also greater in cases vs controls. There were 32,780 nonrepeated interventions in cases vs 7069 in controls. The number of repetitive interventions in cases was $529(1.6 \%)$ and in controls was $748(10.6 \%)$. The interventional spillover effect in cases was $98.4 \%$ vs $89.4 \%$ in controls $(P=0.01)$. The actual interventional spillover effect identified in this case-control study was $9.0 \%$.

\section{Discussion}

To our knowledge, this study is the first of its kind in research methodology to be carried out to evaluate the physician spillover effect in a large RDUR program. Our hypothesis was that the interventions made by the RUDR pharmacists would have an impact on future physician prescribing, and the intervention spillover effect would significantly improve vs the control group. The methodology used to achieve this objective was a case-control study.

We found that the physician spillover effect was greater in cases compared with controls. A physician who was part of a case group was eight times less likely to receive the repeated interventions during the study period than a physician in the control group. The probable cause of high physician spillover

Table I Number of nonrepetitive interventions per prescriber

\begin{tabular}{lll}
\hline $\begin{array}{l}\text { Number of nonrepetitive } \\
\text { interventions }\end{array}$ & Cases & Controls \\
\hline 1 & $14,210(62.1 \%)$ & $930(39.6 \%)$ \\
$2-5$ & $7985(34.9 \%)$ & $1088(46.3 \%)$ \\
$6-10$ & $550(2.4 \%)$ & $249(10.6 \%)$ \\
$11-20$ & $111(0.5 \%)$ & $78(3.3 \%)$ \\
$20+$ & $14(0.1 \%)$ & $3(0.1 \%)$ \\
\hline
\end{tabular}

Table 2 Nonrepetitive conflicts intervened

\begin{tabular}{lll}
\hline $\begin{array}{l}\text { Nonrepetitive } \\
\text { conflict type }\end{array}$ & $\begin{array}{l}\text { Cases } \\
(\mathbf{N}=\mathbf{3 2 , 7 8 0 )}\end{array}$ & $\begin{array}{l}\text { Controls } \\
\mathbf{( N = 7 0 6 9 )}\end{array}$ \\
\hline Therapeutic appropriateness & $8277(25.3 \%)$ & $1117(15.8 \%)$ \\
Therapeutic duplication & $5641(17.2 \%)$ & $1096(15.5 \%)$ \\
Underuse precaution & $6076(18.5 \%)$ & $1057(15.1 \%)$ \\
Drug-drug interaction & $5823(17.8 \%)$ & $1796(25.4 \%)$ \\
High dose & $2703(8.2 \%)$ & $684(9.7 \%)$ \\
Drug-disease precaution & $2347(7.2 \%)$ & $504(7.1 \%)$ \\
Overuse precaution & $1913(5.8 \%)$ & $815(11.5 \%)$ \\
\hline
\end{tabular}

effect in cases was the continuous pharmacist educational interventions during and prior to the study period. The influence of the pharmacist on physician prescribing reduces the need for continuous intervention on the same criteria. The control group did not show a similar improvement in the need for the repeated interventions.

The overall interventional spillover effect was significantly higher in cases compared with controls. The number of repeated interventions was $67 \%$ higher per physician in the controls in addition to an increase in the number of physicians that were intervened, as discussed previously. The differential effect between the cases and controls can only be attributed to the lessons learned from the previous interventions.

A previous study by Hennessy et $\mathrm{al}^{8}$ on RDUR prescribing errors and clinical outcomes was unable to identify an effect of RDUR on the rate of exceptions or clinical outcomes. However, there are many limitations to their study such as the fact that they did not include a comparison control group. The study only took into consideration drug-drug, drugdisease, and duplication criteria. There was much variation in the rate of alerts based on the exceptions generated through their DUR software. Their clinical outcomes assessment is based on the rate of exceptions, and not the rate of alerts, sent to the physician. Also, they did not identify any false positive exceptions which could have impacted the outcome of the study. Finally, they did not check any individualized physician prescribing behavior.

This study has a number of strengths, such as studying the impact of RDUR interventions on actual physician prescribing, looking at all therapeutic categories, and comparing the interventional effect in cases vs controls. All conflicts were assigned a severity score, and interventions were only made in patients with high severity scores that were considered clinically significant. The control group was matched to the case group in terms of severity score. To eliminate any bias, we removed all case physicians from the control group. Additionally, our analysis was based on actual interventions sent to the physician, which directly correlates to the outcomes. 
We did not correlate our interventions with medical outcomes such as hospitalizations. Also, we did not check whether the physician changed the prescription based on the recommendation of the pharmacist. We did not have any baseline data prior to the implementation of the RDUR program in the case and control group. Another limitation to this study is that we assumed that any intervention made on behalf of a patient is the reason that the intervention does not occur again. It could be that the reason a repeated intervention does not occur is that the same factors that caused the first intervention did not occur again, for example a specific drug-drug interaction.

These results indicate that continuous educational interventions by pharmacists can have a significant impact on physician prescribing. Future research should focus on correlating the interventional spillover effect to clinical health outcomes such as hospitalization and emergency room visits. An improvement in health outcomes should lead to reduction in morbidity and mortality in this population. Other areas of research include pharmacoeconomics analysis to determine the cost-benefit of these RDUR programs. A previous study focused on the rates of hospitalizations per exception. ${ }^{8}$ However, it may be more appropriate to look at the direct impact of these RDUR programs by measuring the hospitalizations avoided in the specific populations studied and then extrapolating that to a similar population. Reducing hospitalization even a small amount may justify implementing these programs.
In conclusion, our case-control study which analyzed the impact of RDUR pharmacist interventions on physician prescribing found that physician spillover effect in cases was eight times higher than controls. On average, each case physician received $67 \%$ less interventions compared with controls.

\section{Disclosure}

The authors report no conflicts of interest in this work.

\section{References}

1. Novartis Pharmacy Benefit Report: 2006 Facts and Figures. East Hanover, NJ: novartis; 2006.

2. McLachlan G, editor. A Question of Quality? roads to assurance in medical care. Oxford University Press; 1976.

3. Duncan A. Quality assurance - what now and what next? BMJ. 1980;280:300-302.

4. Angalakuditi MV. Evaluation of pharmacist interventions on drug and dosage prescribing in pediatric settings. Curtin University of Technology PhD Thesis. Perth, Australia 2003. Available from: http://adt.curtin.edu. au/theses/available/adt-WCU20041111.094908/. Accessed June 15, 2011.

5. Knapp DA, Brandon BM, West S, Leavitt DE. Drug use review a manual system. JAm Pharm Assoc. 1973;13:417-421.

6. Palumbo FB, Knapp DA, Brandon BM, Knapp DE, Solomon DK, Klein LS. Detecting prescribing problems through drug usage review: a case study. Am J Hosp Pharm. 1977;34:152-154.

7. Berbatis CG. Strategies to improve drug use in hospitals. Aust Health Rev. 1984;7:253-259.

8. Hennessy S, Bilker WB, Zhou L, et al. Retrospective drug utilization review, prescribing errors, and clinical outcomes. JAMA. 2003;290: 1494-1499.
ClinicoEconomics and Outcomes Research

\section{Publish your work in this journal}

ClinicoEconomics \& Outcomes Research is an international, peerreviewed open-access journal focusing on Health Technology Assessment, Pharmacoeconomics and Outcomes Research in the areas of diagnosis, medical devices, and clinical, surgical and pharmacological intervention. The economic impact of health policy and health systems

\section{Dovepress}

organization also constitute important areas of coverage. The manuscript management system is completely online and includes a very quick and fair peer-review system, which is all easy to use. Visit http://www.dovepress.com/testimonials.php to read real quotes from published authors. 